Journal of Hard Tissue Biology 26[1] (2017) 67- 74 (C) 2017 The Hard Tissue Biology Network Association Printed in Japan, All rights reserved. CODEN-JHTBFF, ISSN 1341-7649

\title{
Original
}

\section{Bone Quality Improvement in Ovariectomized Rats Dosed with New Osteoporosis-Preventive Compound: Analyses with Raman Spectroscopy and Polarized Light Microscopy}

\author{
Hiroshi Nakada, ${ }^{1,5}$, Toshiro Sakae ${ }^{2)}$, Takahiro Takahashi' ${ }^{1)}$, Kanami Sato ${ }^{3)}$, Takehiro Watanabe ${ }^{1,5)}$, Hiroki Sato ${ }^{3)}$, \\ Daisuke Isaji $^{3)}$, Akira Yanagawa ${ }^{4}$, Norio Yasuda), Shukichi Ochiai ${ }^{4)}$, Nobby Kitagawa ${ }^{4)}$, Hiroyuki Okada ${ }^{2,5)}$, \\ Atsuko Gunji ${ }^{1,5)}$, Morio Iijima ${ }^{1,5)}$, Suguru Kimoto ${ }^{1,5)}$ and Yasuhiko Kawai ${ }^{1,5)}$ \\ 1) Department of Removable Prosthodontics, Nihon University School of Dentistry at Matsudo, Matsudo, Japan \\ ${ }^{2)}$ Department of Histology, Nihon University School of Dentistry at Matsudo, Matsudo, Japan \\ 3) Nihon University Graduate School of Dentistry at Matsudo, Matsudo, Japan \\ 4) S.T. Japan Inc, Tokyo, Japan. \\ 5) Research Institute of Oral Science, Nihon University School of Dentistry at Matsudo, Matsudo, Japan \\ (Accepted for publication, November 28, 2016)
}

\begin{abstract}
In this study, to observe the influence of Additive Formula Diet (AFD) ingestion in ovariectomized rats (OVX), OVX were fed a normal mineral diet (NMD) or AFD, and structures of the outer and inner regions of the cross-section of the central region of the femoral diaphysis and changes in crystalline bone quality were investigated by polarized light microscopy and Raman spectroscopy. For experimental animals, 8 rats ovariectomized at 20 weeks old were used. OVX were divided into 2 groups: NMD-fed ONMD group and AFD-fed OAFD group, and the femur was excised 24 weeks after intervention. On polarized light microscopy, the external basic lamella was thicker in the OAFD than ONMD group, showing higher-order colors. On Raman spectroscopy, the degree of calcification and crystallinity in the inner region tended to slightly higher in the OAFD than ONMD group, although crystallinity tended to slightly lower. Ingestion of AFD may have improved bone metabolism throughout the body, and improvement of the femoral mineral levels and collagen cross linkages may have more markedly improved bone quality compared with that by NMD ingestion, suggesting its usefulness for treatment of osteoporosis, bone healing after tooth extraction, and early bone formation after implant placement.
\end{abstract}

Key words: Raman Spectroscopy, Osteoporosis, Supplement, Bone quality, Ovariectomized rat

\section{Introduction}

There are about 75 million osteoporosis patients worldwide ${ }^{1)}$, and the number is increasing yearly. Osteoporosis was previously considered as a mere aging phenomenon, but it is an important disease in which the condition painlessly progresses with time to a morbid bone state and affects patient's quality of life. The major problem of osteoporosis patients is fracture due to fall and the patient becomes bed-ridden. When vertebral compression fracture occurs, daily living activities are markedly reduced; therefore, early diagnosis, treatment, and prevention are important. Fractures in osteoporosis patients occur due to reduced bone strength, i.e., bone fragility caused by deterioration of bone quality and reduction

Correspondence to: Dr. Hiroshi Nakada, Department of Removable Prosthodontics, Nihon University School of Dentistry at Matsudo, 2-8701 Sakaecho-Nishi, Matsudo, Chiba, 271-8587, Japan; Phone: +81-47360-9379; Fax: +81-47-360-9376; E-mail: nakada.hiroshi@nihon-u.ac.jp of bone mineral density (BMD). For drug treatment of osteoporosis, a parathyroid hormone preparation, teriparatide, is used. Parathyroid hormone elevates the blood calcium level by promoting bone resorption, for which a constantly high blood calcium level is necessary, and enhancement of bone metabolism is also necessary. Thus, it may be necessary to always have a readily ingestible supplement and combine it with a drug for treatment of osteoporosis.

Experiments on osteoporosis are performed using experimental animal models of osteoporosis prepared by excision of the ovaries. It has been reported that changes in bone induced by reduced secretion of a female hormone, estrogen, similar to those in humans, occur in experimental osteoporosis animal models ${ }^{2)}$. Experimental ovariectomized rats (OVX) are used in many studies on changes in systemic bone metabolism ${ }^{3-7)}$. We fed a fructooligosaccharide-, isoflavone-, and calcium phosphate- 
compounded osteoporosis-preventive supplemental feed (High mineral diet (HMD): calcium compounding ratio 3.0\%) to OVX and observed changes in bone quality ${ }^{6,7)}$. We reported that feeding HMD to OVX for 24 weeks was useful to improve bone quality of the femoral diaphysis by increasing BMD and breaking strength ${ }^{6}$. However, although the compounded calcium level, $3.0 \%$, in HDM was useful to improve bone quality, the mineral intake became excessive for the body, and it may upset the nutrient balance $^{6)}$. To improve HMD, we prepared a new triple compound feed, Additive Formula Diet (AFD), in which the calcium source was changed to calcium citrate, which is more soluble than calcium phosphate ${ }^{8,9)}$, and the calcium level was adjusted to a level similar to that in general commercial feeds (compounding ratio: $1.0 \%$ ). AFD was prepared as a supplement to prevent osteoporosis and improve the blood mineral level, bone metabolism, and bone quality after surgical treatment of bone, such as dental implant treatment.

Bone is the largest calcium storage organ in the body, and it is also a regulatory organ maintaining homeostasis. In bone metabolism, calcium is one of the most important minerals for the body, and its extracellular and intracellular levels are strictly controlled for normal cell functions, maintaining homeostasis. Bone metabolism also plays an important role in adjusting blood levels of not only calcium but also other minerals, such as phosphorus and magnesium. Hardness and fragility of bone tissue are determined by the mineral content in bone ${ }^{10)}$, which increases with aging ${ }^{11,12)}$, but it gradually decreases after 50 years old ${ }^{13)}$. Changes in the mineral composition are related to the size and distribution of microcrystals or molecular-structural substitution (e.g., $\mathrm{Na}^{+}, \mathrm{K}^{+}, \mathrm{F}^{-}, \mathrm{Mg}^{2+}, \mathrm{CO}_{3}{ }^{2-}$ ), and tissue structural changes include changes in collagen cross linkages ${ }^{12,14)}$. It has been reported that differences in the properties among bone types and mechanical properties markedly vary in adults, whereas age- and sex-related differences are small ${ }^{15,16)}$. Bone strength depends on not only the degree of calcification but also mineral crystallinity, quality of calcium phosphate crystals, and compounded components ${ }^{17)}$. In addition, structural changes in collagen cross linkages are as important as the mineral content to improve bone quality, and homogenous deposition of minerals in collagen may be necessary to acquire strong bone quality. A correlation between fractureinduced material properties of tissue and collagen cross linkage has been reported ${ }^{18)}$, but when the amount and quality of collagen change, minerals cannot readily deposit on fibers, i.e., even though the content of minerals including calcium is sufficient, if the quality of collagen cross linkage structures is poor, the bone quality is also poor. Morris MD et al. reported various studies performed extensively from the clinical concept to reviews using Raman spectroscopy, which is used to measure both indices: minerals and collagen ${ }^{19-21)}$.

Raman spectroscopy has a micro-level spatial resolution and
Table 1. Compositions of the normal mineral diet (NMD) and the additive formula diet (AFD).

\begin{tabular}{lll}
\hline Composition & NMD & AFD \\
\hline$\alpha$-Cornstarch & 42.87 & 31.37 \\
$\beta$-Cornstarch & 15.50 & 15.50 \\
Casein & 14.00 & 14.00 \\
L-Cystine & 0.18 & 0.18 \\
Sucrose & 10.00 & 10.00 \\
Soybean Oil & 4.00 & 4.00 \\
Cellulose Powder & 5.00 & 5.00 \\
Mineral Mixture & 3.50 & 3.50 \\
Vitamin Mixture & 1.00 & 1.00 \\
Choline Bitartrate & 0.25 & 0.25 \\
Tert-Butylhydroquinone & 0.00 & 0.00 \\
Additives & & \\
Calcium carbonate & 0.90 & \\
Fructooligosaccharides & & $\mathbf{1 0 . 0 0}$ \\
Isoflavone & & $\mathbf{0 . 5 0}$ \\
Calcium citrate tetrahydrate & & $\mathbf{3 . 0 0}$ \\
Calcium phosphate, Dibasic & 2.80 & 1.70 \\
\hline Total \% & 100.00 & 100.00 \\
\hline Ca & $\mathbf{1 . 0 1}$ & $\mathbf{1 . 0 3}$ \\
$\boldsymbol{P}$ & $\mathbf{0 . 6 0}$ & $\mathbf{0 . 6 1}$ \\
\hline
\end{tabular}

it allows qualitatively and quantitatively analysis of molecular structural changes in collagen and hydroxyapatite. A microscope equipped with an auto-stage is capable of imaging. The following bone qualities can be evaluated using the main Raman bands of bone, collagen, and hydroxyapatite ${ }^{19-21)}$ : Phosphate/Amide I ratio (degree of calcification), Carbonate/Phosphate ratio (indirect scale of remodeling activity), and Carbonate/Amide I ratio (bone reformation). These intensity ratios can be used to evaluate bone quality. Regarding crystallinity of hydroxyapatite, the full-width half-maximal (FWHM) of the Phosphate band $\left(\mathrm{PO}_{4}{ }^{3-}\right)$ is used in many studies ${ }^{22-23)}$. FWHM is an important index related to modulus of elasticity, yield stress, and breaking stress of bone. In addition, the Phosphate/Amide I ratio and Carbonate/Amide I ratio are indices of mechanical property.

The details of bone crystallinity, remodeling activity, or bone reformation in AFD-fed OVX have not been analyzed based on chemical composition from the viewpoint of degree of mineral calcification, crystallinity, or maturation degree of bone matrix. Thus, to observe the influence of AFD ingestion in OVX, the influences on the inner region contacting the bone marrow and outer region were compared in the cross-section of the central region of the femoral diaphysis, in which blood flow is closely involved in bone metabolism. In this study, OVX was fed a normal mineral diet (NMD) or AFD, and changes in the structures of the inner and outer regions and crystalline bone qualities were 


\section{a) Femur mid-shaft region of ONMD group (left), enlarged image (right)}
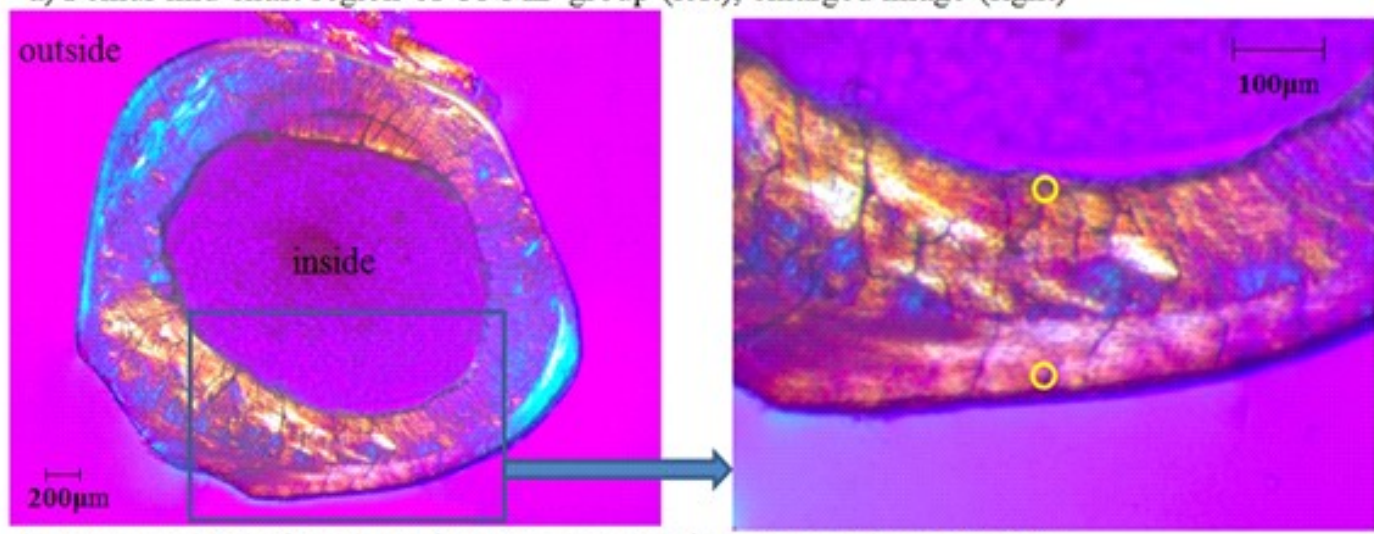

b) Femur mid-shaft region of OAFD group (left), enlarged image (right)

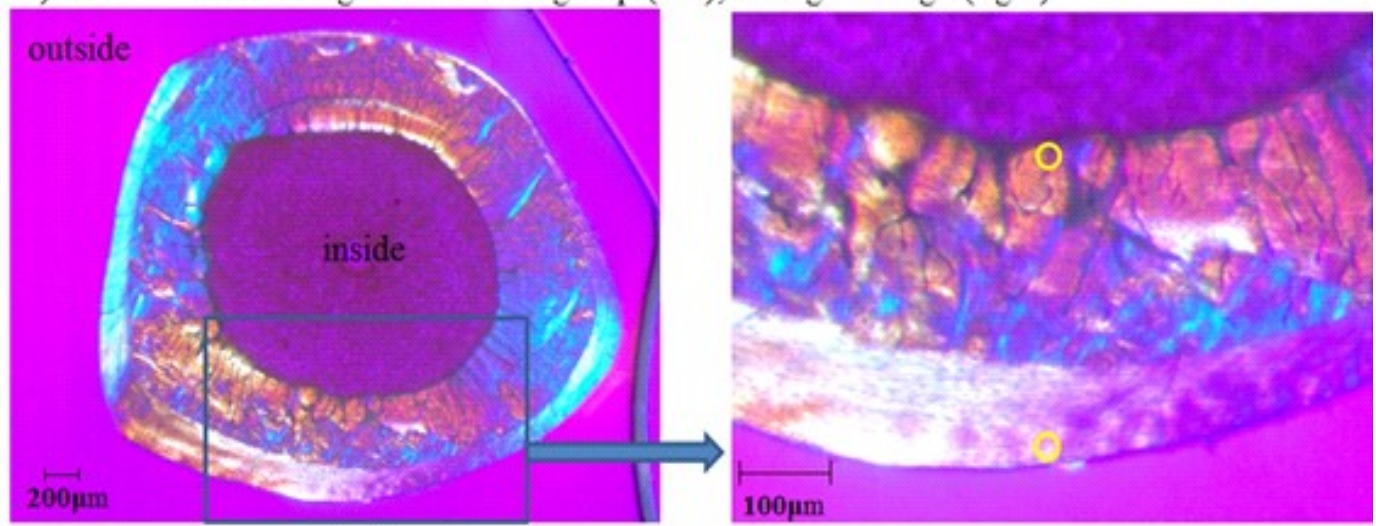

Figure 1. Cross-sectional views of the center of the femoral diaphysis on polarized light microscopy in the ONMD and OAFD groups. The left pictures show images at low magnifications and, the right pictures show images at high magnifications. The analyzing spots of Raman spectroscopy were the center of the circle (right picture).

investigated in the femur by Raman spectroscopy and polarized light microscopy.

\section{Materials and Methods \\ Experimental animals}

For experimental animals, 8 female Wistar rats aged 20 weeks were used. Rats that received bilateral ovariectomy at 20 weeks old were purchased from Sankyo Labo Service Corporation, Inc. (Tokyo, Japan) as an osteoporosis model. Four animals were randomly selected to establish 2 groups. OVX were fed NMD (AIN-93M, Oriental Yeast Co., Ltd., Tokyo, Japan; ONMD group) or AFD (Oriental Yeast Co., Ltd., Tokyo, Japan; OAFD group) for 24 weeks. The compositions of NMD and AFD feeds are shown in Table 1. All OVX were euthanized using carbonate gas 24 weeks after initiation of feeding, and the right femur was excised. This study was performed after approval of the Animal Experiment Committee of Nihon University School of Dentistry at Matsudo (approval number: AP11MD023).

\section{Preparation of Non-decalcified Specimens for Raman Spectroscopy}

The femur was dehydrated and defatted in a $70-100 \%$ alcohol series, and embedded in resin using the Osteoresin Embedding Kit (Wako Pure Chemical Industries, Osaka, Japan). After embedding, $30-\mu \mathrm{m}$ non-decalcified cross-sections of the middle region of the femur were prepared using an automatic highprecision cutter (Buller, Illinois, USA). These non-decalcified sections were not stained, because any staining dyes affect the resulted Raman Spectrum and conceal the initial pattern of the specimen.

\section{Polarized light microscopic observation of femurs}

In polarized light microscopic observation, changes in the inside and outside regions of the femur were observed by the gypsum plate method using Leica M60 (Leica Microsystems GmbH, Wetzlar, Germany).

\section{Raman spectroscopy}

On Raman spectroscopy, spectra of the inner and outer regions of the femur were measured at a randomly selected site in each sample. The analyzing spots was the center of the circle (Fig. 1). The device used was a RAMAN RXN1 Microprobe (Kaiser Optical Systems Inc., Michigan, USA); measurement software, HoloGRAMS version 4.1 (Kaiser Optical Systems Inc., Michigan, 
Table 2. FWHM and peak ratio in each Raman property (mean \pm $\mathrm{SD})$ between ONMD and OAFD groups for inner and outer regions of femurs. Student's T-test, $\mathrm{n}=4, *: p<0.05$

\begin{tabular}{lcc}
\hline & ONMD - inside & ONMD - outside \\
\hline $\mathrm{PO}_{4}{ }^{3-}$ (FWHM) & $18.00 \pm 0.00$ & $18.00 \pm 0.00$ \\
Phosphate / Amide I & $1.82 \pm 0.12$ & $2.08 \pm 1.19$ \\
Carbonate / Phosphate & $0.12 \pm 0.01$ & $0.16 \pm 0.02 *$ \\
Carbonate / Amide I & $0.22 \pm 0.02$ & $0.31 \pm 0.16$ \\
\hline & & \\
& OAFD - inside & OAFD - outside \\
\hline $\mathrm{PO}_{4}{ }^{3-}$ (FWHM) & $18.33 \pm 0.58$ & $18.00 \pm 0.00$ \\
$\mathrm{Phosphate}^{*}$ Amide I & $2.62 \pm 0.21$ & $2.21 \pm 0.21$ \\
Carbonate / Phosphate & $0.14 \pm 0.01$ & $0.14 \pm 0.02$ \\
Carbonate / Amide I & $0.38 \pm 0.05$ & $0.30 \pm 0.07$ \\
\hline
\end{tabular}

Student's T-test, $\mathrm{n}=4, *$, $\mathrm{p}<0.05$

USA), spectral data processing; GRAMS / AI (Thermo Fisher Scientific Inc., Massachusetts, USA) and Panorama (LabCognition Analytical Software GmbH \& Co. KG, Cologne, Germany), excitation laser wavelength, $785 \mathrm{~nm}$; laser exposure time, 10 seconds; cumulative frequency, 6; and spatial resolution, 2ìm. For the femoral specimens, the integrated intensities of 3 characteristic bands were measured: phosphate ( $\mathrm{PO}_{4}^{3-}$; ca. 903-991 $\left.\mathrm{cm}^{-1}\right)$, carbonate ( $\mathrm{CO}_{3}^{2-;}$ ca. 1046-1110 $\mathrm{cm}^{-1}$ ), and matrix collagen amide I (ca. 1616-1720 $\mathrm{cm}^{-1}$ ). Ratios of these band areas resulted in measures of Carbonate/Phosphate,1 Phosphate/Amide I and Carbonate/Amide I. The full-width half-maximal (FWHM) of the phosphate band was measured to evaluate mineral crystallinity. The ratios were calculated on each peak, and then averaged for each specimen, resulting in a single value of each ratio for each specimen. FWHM and integrated intensity were measured and evaluated following reports of McCreadie BR et al. ${ }^{18)}$ and Morris MD et al. ${ }^{19)}$.

\section{Statistical analysis}

For between-group comparison of the inner and outer regions of the femur, Student's t-test was employed, and $\mathrm{p}<0.05$ was regarded as significant. All statistical analyses were performed using the statistical package PASW Statistics (Version 22.0, SPSS, Chicago, IL, USA).

\section{Results}

\section{Observation of femurs}

The entire and magnified views of the femur in the ONMD and OAFD groups on polarized light microscopy are shown in Fig. 1. The polarization property representing characteristic orientation of collagen fibers was observed in both groups. In the entire view, the cortical bone width was thicker in the OAFD than ONMD group. In the magnified view, many thicker tubular structures of Volkmann's canal were observed in the inner region in the OAFD than ONMD group, showing favorable development. Haversian lamella with higher-order colors presented in blue was observed in the middle of the cortical bone width in the OAFD group, but it was scattered in the ONMD group. In addition, the

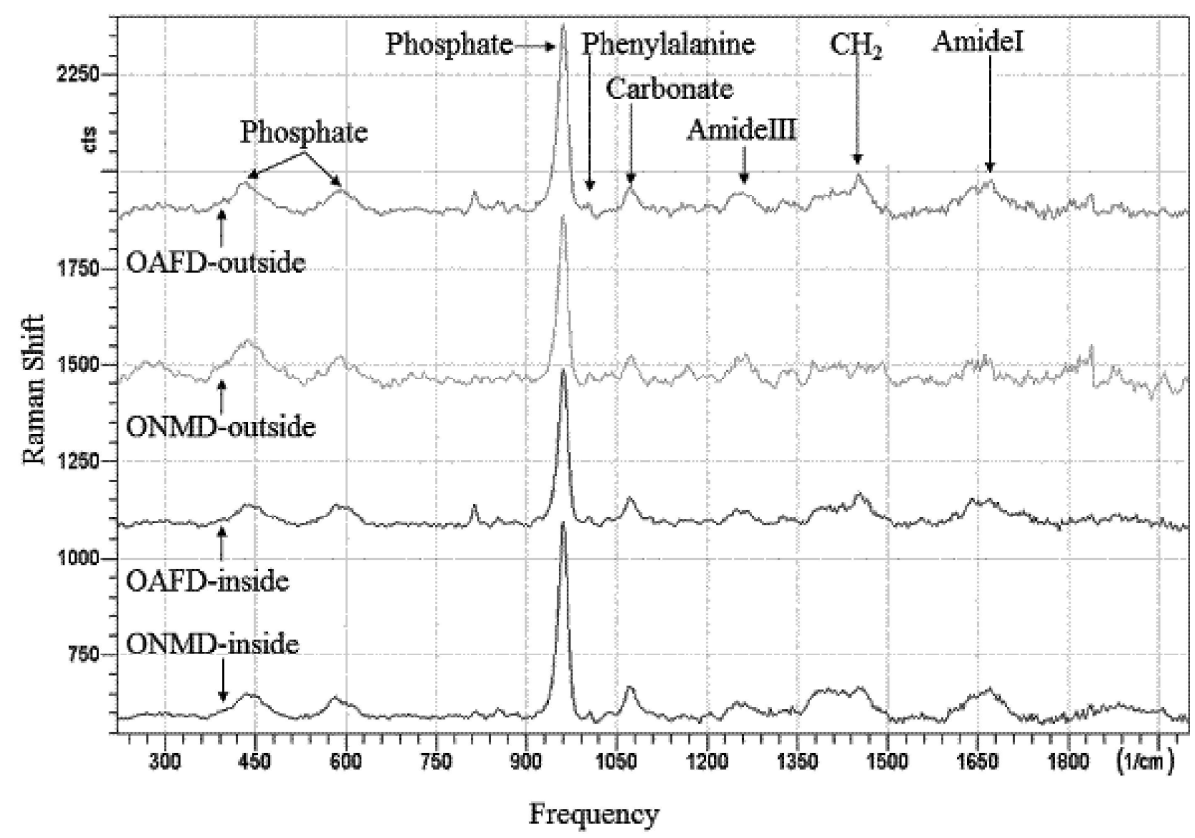

Figure 2. Ramen spectra of the inner and outer regions of the femur in the ONMD and OAFD groups. From the bottom, ONMD group-inner region, OAFD group-inner region, ONMD group-outer region, and OAFD group-outer region. Typical functional groups are presented. 

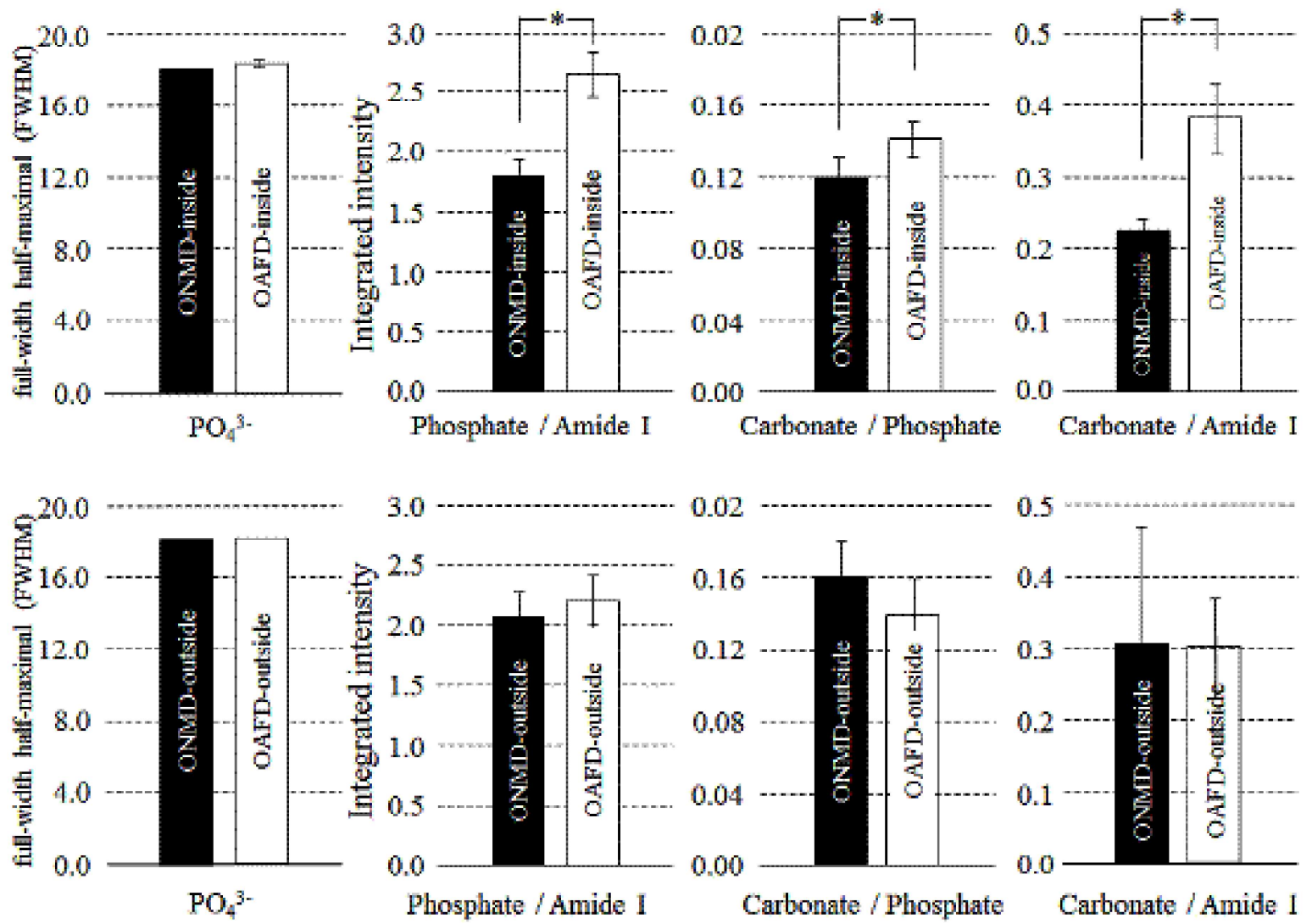

Student's T-test, $\mathrm{n}=4, p<0.05$

Figure 3. FWHM and peak ratio in each Raman property (mean \pm SD) between inner and outer regions of femurs for ONMD and OAFD groups. Student's T-test, $\mathrm{n}=4, *: p<0.05$

external basic lamella was thicker in the OAFD than ONMD group, showing higher-order colors.

\section{Raman spectroscopy}

The Raman spectroscopy spectra of the inner and outer regions of the femur in the ONMD and OAFD groups are shown in Fig. 2. Peaks of hydroxyapatite-related functional groups were detected in the 4 spectra. $\mathrm{PO}_{4}^{3-}$ (FWHM), Phosphate/Amide I, Carbonate/ Phosphate, and Carbonate/Amide I were measured in the typical spectra of the ONMD and OAFD groups shown in Fig. 2. The results are shown in Table 2 and Fig.3.

In the ONMD group, no change was noted in $\mathrm{PO}_{4}^{3-}$ (FWHM) in the inner or outer region, whereas Phosphate/Amide I and Carbonate/Amide I tended to slightly higher in the outer than inner region, and Carbonate/Phosphate was significantly higher in the outer region, showing that the degree of calcification and carbonate content rate of hydroxyapatite were high in the outer region in the femur in the ONMD group. On comparison between the inner and outer regions in the OAFD group, $\mathrm{PO}_{4}^{3-}$ (FWHM), Phosphate/ Amide I, and Carbonate/Amide I tended to slightly higher in the inner region, indicating that the degree of calcification was higher and crystallinity tended to slightly lower in the inner than outer region of the femur. The comparison in the inner and outer regions of the ONMD group and the OAFD group is shown in Fig.3. On comparison between the ONMD and OAFD groups in the inner region, $\mathrm{PO}_{4}^{3-}$ (FWHM) tended to slightly higher in the OAFD group, and Phosphate/Amide I, Carbonate/Phosphate, and Carbonate/Amide I were significantly higher in the OAFD group. Phosphate/Amide I in the outer region (Fig.3) tended to slightly higher in the OAFD group, but $\mathrm{PO}_{4}^{3-}$ (FWHM), Carbonate/ Phosphate, and Carbonate/Amide I tended to similar. Therefore, on comparison of the outer region of the femur, no significant difference was noted in the crystallinity, degree of calcification, or carbonate content rate. However, on comparison of the inner region of the femur, the crystallinity tended to slightly lower in the OAFD than ONMD group, but the degree of calcification and carbonate content rate tended to slightly higher in the OAFD group.

\section{Discussion}

On polarized light microscopy, the modification of the bone structure was promoted from the inner wall of cortical bone in the OAFD group compared with that in the ONMD group, and development of Volkmann's canal was clearly observed, suggesting that mineral components diffused over the bone from the medullary space through Volkmann's canal. Collagen fibers and bone minerals showed intrinsic double refractions on polarized light 


\section{J.Hard Tissue Biology Vol. 26(1): 67- 74, 2017}

microscopy. Since double refraction of collagen fibers is stronger than that of bone minerals, double refraction is mainly noted in the direction of collagen fiber arrangement. Differences in the sites and number of double refractions were noted between the OAFD and ONMD groups, and collagen fiber arrangement and maturation activity of bone quality increased.

On Raman spectroscopy, calcification, remodeling activity, and bone reformation were enhanced in the outer than inner region in the femoral diaphysis in the ONMD group. In OVX used in this study, estrogen secretion is decreased by ovariectomy and osteoclast activity becomes dominant over osteoblast activity, through which bone resorption surpasses bone formation ${ }^{24}$. Remodeling induced by reduced estrogen secretion causes perforation of cancellous trabeculae, and the trabecular width decreases due to rupture of pillar structures and the number of the pillar structures decreases, changing the bone structure and reducing bone quality. Khodadadyan et al. measured the cortical bone width and observed that it decreased by $7.0 \%$ in OVX 6 months after ovariectomy ${ }^{25}$. In addition, osteoclast activity became dominant over osteoblast activity in the inner wall of cortical bone due to reduction of estrogen secretion in OVX, and we previously reported that bone resorption markedly decreased the cortical bone width $^{26)}$. Salomon et al. found that cortical bone is markedly resorbed from Haversian canal and the inner wall of micro lumens of Volkmann's canal ${ }^{27)}$, and thinning of cortical bone is consistent with that observed in our previous study ${ }^{26)}$. The bone width was thinner in the ONMD than OAFD group on polarized light microscopy, showing a similar finding. It was suggested by Raman spectroscopy that the degree of calcification based on Phosphate/ Amide I, remodeling activity based on Carbonate/Phosphate, and bone reformation based on Carbonate/Amide I tended to higher in the outer than inner region in the ONMD group, indicating improvement of bone quality, and accordingly, bone quality may have been reduced in the inner region compared with that in the outer region. In the OAFD group, crystallinity tended to slightly higher in the inner than outer region, but the degree of calcification and bone reformation were high, suggesting improvement of bone quality. On comparison of the inner region between the ONMD and OAFD groups, the degree of calcification, and remodeling activity and bone reformation levels were high in the OAFD group, suggesting improvement of bone quality. On comparison of the outer region, it was suggested that bone quality was similar between the ONMD and OAFD groups.

Fructooligosaccharide compounded in AFD feed is distributed to bone throughout the body through mineral absorption from the intestine and subsequent circulation, and it has been reported to increase BMD and bone mineral content and improve bone quality $^{28)}$. In addition, isoflavone is structurally similar to estrogen and it has estrogen-like action with affinity for estrogen receptors, ERá and $\mathrm{ERa}^{29)}$. Furthermore, isoflavone inhibits bone-resorption cytokines, which directly act on bone, and facilitates inhibition of bone resorption by dominant osteoclasts induced by reduced estrogen secretion. Calcium citrate is a readily absorbed calcium component compared with calcium carbonate ${ }^{8,9)}$. Since it is watersoluble and binds to acid, it is readily absorbed into the body through chelating action. Therefore, the 3 components contained in AFD were absorbed from the intestine and the mineral components were distributed throughout the body through the circulation, which may have increased bone quality from the inner region contacting the bone marrow. In the femur, Haversian lamella and Volkmann's canal may have distributed blood flow from the inner region of cortical bone contacting the bone marrow to the outer region, improving and maintaining bone quality. The findings in the inner region in the 2 groups suggest that, even in the same regions with good blood flow, blood flow containing abundant mineral components from ADF feed improved bone metabolism and bone quality in the OAFD group, compared with that in the ONMD group.

Evaluation of bone quality is influenced not only by breaking strength but also various factors such as calcium phosphate crystallinity, degree of calcification, remodeling activity level, and bone reformation. For evaluation of superiority or inferiority of bone quality, structural changes in collagen cross linkages are important, and homogeneous mineral deposition in collagen is necessary to acquire strong bone quality. Therefore, ingestion of AFD may have improved systemic bone metabolism, and improvement of the femoral mineral level and collagen cross linkage may have more markedly improved bone quality compared to that by NMD ingestion, suggesting its usefulness for treatment of osteoporosis, bone healing after tooth extraction, and early bone formation after implant placement. Although no in vivo clinical experiment of AFD ingestion has been performed, enhanced bone metabolism improved bone quality, suggesting its usefulness as a preventive and therapeutic drug for osteoporosis.

Osteoporosis cannot be explained only by mineral deficiency, postmenopausal reduction of estrogen secretion, and aging phenomenon, and diverse life-style-related factors may be involved in a complex way. We analyzed the influence of feed on rats with osteoporosis, but the direct influence of each component: calcium, phosphorus, magnesium, zinc, and fluorine, was not analyzed. We will investigate the influences of minerals. We are planning to clarify the details of bone quality such as dynamics of osteoblasts and osteoclasts in the femoral diaphysis, collagen cross linkages, and growth of crystals.

\section{Acknowledgement}

This work was supported in part by KAKENHI; Young Scientists (B) (15H06645: T. Watanabe) from the Japan Society for the Promotion of science 
Hiroshi Nakada et al.: Bone changes in OVX rats

\section{Competing Interests}

I have the following collaboration research to disclose. Device of Raman spectroscopy used in this study was provided by S.T. Japan Inc. Tokyo, Japan.

We ask S.T. Japan Inc. to analyze bone with Raman spectroscopy without conflict of interest.

\section{References}

1. WHO Scientific Group. Prevention and Management of Osteoporosis, World Health Organization, Geneva, 2003

2. Zaidi M. Skeletal remodeling in health and disease. Nat Med 13: 791-801, 2007

3. Wronski TJ, Dann LM, Scott KS and Cintrón M. Longterm effects of ovariectomy and aging on the rat skeleton. Calcif Tissue Int 45: 360-366, 1989

4. Jiang GZ, Matsumoto H, Hori M, Gunji A, Hakozaki K, Akimoto $\mathrm{Y}$ and Fujii A. Correlation among geometric, densitometric, and mechanical properties in mandible and femur of osteoporotic rats. J Bone Miner Metab 26: 130137, 2008

5. Nakada H, Suzuki S, Sakae T, Tanimoto Y, Kuboyama N, Teranishi M, Kato T, Watanabe T, Kimura-Suda H, LeGeros RZ and Kawai Y. Quantitative and qualititative analyses of low-mineral-diet ovariectomised rat femora using microscopic computed tomography. J Hard Tissue Biol 20: 107-114, 2011

6. Nakada H, Sakae T, Watanabe T, Takahashi T, Fujita F, Tanimoto Y, Okada H, Kaneda K, Kato T and Kawai Y. A new osteoporosis prevention supplements-diet improve bone mineral density in ovariectomized rats on micro-CT. J Hard Tissue Biol 23: 1-8, 2014

7. Nakada H, Sakae T, Watanabe T, Takahashi T, Fujita F, Tanimoto Y, Okada H, Kaneda K, Kato T and Kawai Y. Structure model index changes in the femoral epiphyseal region on micro-computed tomography caused by a supplement diet in ovariectomized rats. J Hard Tissue Biol 23: 169-176, 2014

8. Nicar MJ and Pak CY. Calcium bioavailability from calcium carbonate and calcium citrate. J Clin Endocrinol Metab 61: 391-393, 1985

9. Harvey JA, Zobitz MM and Pak CY. Dose dependency of calcium absorption: a comparison of calcium carbonate and calcium citrate. J Bone Miner Res 3: 253-258, 1988

10. Currey JD. The effect of porosity and mineral content on the Young's modulus of elasticity of compact bone. J Biomech 21: 131-139, 1988

11. Paschalis EP, Shane E, Lyritis G, Skarantavos G, Mendelsohn $\mathrm{R}$ and Boskey AL. Bone fragility and collagen cross-links. J Bone Miner Res 19: 2000-2004, 2004
12. Hernandez CJ, Tang SY, Baumbach BM, Hwu PB, Sakkee AN, van der Ham F, DeGroot J, Bank RA and Keaveny TM. Trabecular microfracture and the influence of pyridinium and non-enzymatic glycation-mediated collagen cross-links. Bone 37: 825-832, 2005

13. Crofts RD, Boyce TM and Bloebaum RD. Aging changes in osteon mineralization in the human femoral neck. Bone 15: 147-152, 1994

14. Ager JW, Nalla RK, Breeden KL and Ritchie RO. Deepultraviolet Raman spectroscopy study of the effect of aging on human cortical bone. J Biomed Opt 10: 034012, 2005

15. Choi K, Kuhn JL, Ciarelli MJ and Goldstein SA. The elastic moduli of human subchondral, trabecular, and cortical bone tissue and the size-dependency of cortical bone modulus. J Biomech 25: 1371-1381, 1990

16. Hoffler CE, Moore KE, Kozloff K, Zysset PK and Goldstein SA. Age, gender, and bone lamellae elastic moduli. J Orthop Res 18: 432-437, 2000

17. Boskey A. Bone mineral crystal size. Osteoporos Int 14 (Suppl 5): S16-S21, 2003

18. McCreadie BR, Morris MD, Chen TC, Sudhaker Rao D, Finney WF, Widjaja E and Goldstein SA. Bone tissue compositional differences in women with and without osteoporotic fracture. Bone 39: 1190-1195, 2006

19. Morris MD and Mandair GS. Raman assessment of bone quality. Clin Orthop Relat Res 469: 2160-2169, 2011

20. Carden A and Morris MD. Application of vibrational spectroscopy to the study of mineralized tissues (review). J Biomed Opt 5: 259-268, 2000

21. Awonusi A, Morris MD and Tecklenburg MM. Carbonate assignment and calibration in the Raman spectrum of apatite. Calcif Tissue Int 81: 46-52, 2007

22

Akkus O, Adar F and Schaffler MB. Age-related changes in physicochemical properties of mineral crystals are related to impaired mechanical function of cortical bone. Bone 34: 443-453, 2004

23. Penel G, Leroy G, Rey C and Bres E. MicroRaman spectral study of the $\mathrm{PO}_{4}$ and $\mathrm{CO}_{3}$ vibrational modes in synthetic and biological apatites. Calcif Tissue Int 63: 475-481, 1998

24. Khodadadyan-Klostermann C, von Seebach M, Taylor WR, Duda GN and Haas NP. Distribution of bone mineral density with age and gender in the proximal tibia. Clin Biomech (Bristol, Avon) 19: 370-376, 2004

25. Hodgkinson A, Aaron JE, Horsman A, McLachlan MS and Nrodin BE. Effect of oophorectomy and calcium deprivation on bone mass in the rat. Clin Sci Mol Med 54: 439-446, 1978

26. Nakada H, Suzuki S, Watanabe T, Sakae T, Tanimoto Y, Kuboyama N, Nakadai M, Teranishi M, Sakurai H, Kimoto S, Yazaki T, Hayashi Y, Gunji A, Saeki H, Kato T and 
J.Hard Tissue Biology Vol. 26(1): 67- 74, 2017

Kawai Y. Changes in quality of the femoral diaphyseal region in ovariectomised rats with different mineral consumption: observations with micro-CT. Nihon Univ J Oral Sci 37: 13-21, 2011

27. Salomon CD and Volpin G. The effects of calcium-deficient diet on breaking strength and other physical parameters of rat bones. Clin Orthop Relat Res 82: 207-213, 1972

28. Ohta A, Baba S, Ohtsuki M, Taguchi A and Adachi T.
Prevention of coprophagy modifies magnesium absorption in rats fed with fructo-oligosaccharides. Br J Nutr 75: 775784, 1996

29. Ishimi Y, Arai N, Wang X, Wu J, Umegaki K, Miyaura C, Takeda A and Ikegami S. Difference in effective dosage of genistein on bone and uterus in ovariectomized mice. Biochem Biophys Res Commun 11:697-701, 2000 